\title{
Essentials of Breast Lump assessment and Surgery for early breast cancer for Surgeons
}

\author{
Prof Ravi Kant, Prof Bina Ravi \\ Integrated Breast Care Centre, Surgery Department, AIIMS, Rishikesh, Uttarakhand
}

Triple Assessment of a breast mass - must for all surgeons

\section{History and Examination}

A detailed history and examination to be performed by a surgeon. In the history, further to the clinical details of their presenting complaint, any potential risk factors are identified, including family history and current medications. The full breast examination is predominantly focused around breast palpation and assessment of the axillary nodes.

\section{Imaging}

Mammography involves 2 compression views of the breast (oblique and cranio caudal), allowing for the detection mass lesions or microcalcifications.

Ultrasound scanning is more useful in women < 35 years and in men, due to the density of the breast tissue in identifying anomalies. It is also routinely used during core biopsies.

MRI imaging is not used in the mainstay of triple assessment however can be useful in the assessment of lobular breast cancers (and in assessing response to neo adjuvant therapy); whilst it has high sensitivity, it has a low specificity.

3. FNAC/Biopsy - A core biopsy provides full histology (as opposed to fine needle aspiration (FNA) which only provides cytology), allowing differentiation between invasive and in-situ carcinoma. The test can generate important information about tumour grading and staging, and has a higher sensitivity and specificity than FNA for detecting breast cancer.

If a woman has recurrent cystic disease (and the lesion is clinically a cyst), this can be aspirated using FNA at this stage for cytology and to relieve symptoms.

No breast lump to be excised without a triple assessment.

\section{Differential Diagnosis}

At each stage of the triple assessment, the suspicion for malignancy is graded to create an overall risk index, as discussed below. The key here is to establish whether this is likely a benign lesion or whether the patient should go onto have more definitive biopsy and further intervention. 


\section{A gap in quality of healthcare}

Gap exists whenever variability of care coexists. In clinical practice significant variability is seen,as far as assessment and treatment of breast lumps are concerned. At our centre we witness breast masses being mismanaged in smaller centres and in private setup.

Many reports have documented significant variability of care for oncologic reoperation after initial lumpectomy for breast cancer.1, 2 Rates of reoperation vary from less than $10 \%$ to more than $50 \%$. This variability is not accounted for by patient or disease characteristics. Therefore, the American Society of Breast Surgeons (ASBrS ) convened a multidisciplinary consensus conference entitled a ",,Collaborative Attempt to Lower Lumpectomy Reoperation rates ${ }^{\text {ece }}$ (CALLER)3.

They developed a practical toolbox of recommendations to help providers reduce lumpectomy reoperations to the best achievable level based on available evidence and expert opinion.

\section{Tool Box}

Tool 1: Preoperative Diagnostic Imaging Should Include Full-Field Digital Mammography and Supplementary Imaging to Include Ultrasound as Needed

High-quality, meticulous, preoperative, diagnostic mammography is necessary preoperatively. "Selective" use of ipsilateral ultrasound (US) recommended. Screening mammography identifies calcifications without a mass. Routine use of MRI is not recommended based on meta-analyses that show its use does not affect the rate of re excision or local recurrence. Selective use of MRI can be done.

\section{Tool 2: Minimally Invasive Breast Biopsy (MIBB) for Breast Cancer Diagnosis}

Some studies demonstrate lower reoperation rates when a diagnosis of malignancy is known before surgical excision. MIBB provides opportunity for preoperative treatment planning to include genetic risk assessment, medical oncology, and plastic surgery consultation and axillary evaluation.

\begin{tabular}{lll}
\multicolumn{1}{c}{ Examination Score } & \multicolumn{1}{c|}{$\begin{array}{c}\text { Imaging Score (Mammography (M), } \\
\text { Ultrasound (U)) }\end{array}$} & \multicolumn{1}{c|}{ Histology Score } \\
\hline P1 - Normal & M1 / U1 - Normal & B1 - Normal \\
P2 - Benign & M2 / U2 - Benign & B2 - Benign \\
$\begin{array}{l}\text { P3 - Uncertain/likely } \\
\text { benign }\end{array}$ & M3 / U3 - Uncertain/likely benign & $\begin{array}{l}\text { B3 - Uncertain, probably } \\
\text { benign }\end{array}$ \\
$\begin{array}{l}\text { P4 - Suspicious of } \\
\text { malignancy }\end{array}$ & M4 / U4 - Suspicious of malignancy & $\begin{array}{l}\text { B4 - Suspicious of } \\
\text { malignancy }\end{array}$ \\
P5 - Malignant & M5 / U5 - Malignant & B5 - Malignant
\end{tabular}


Tool 3: Multidisciplinary Discussions to Include Radiology, Pathology, Surgery, and Radiation and Medical Oncology if lump is malignant

Optimizing reoperation rates requires preoperative collaboration between radiologists, surgeons, and pathologists. In patients considered for neo adjuvant therapy, medical oncology consultation also is necessary. Preoperative knowledge of number of lesions, geometry, distance to skin and chest wall, and possible extension towards the nipple may all facilitate negative margins. Information technology that enhances communication and provides intraoperative archived images can aid lesion review and communication. Postoperative discussion with all specialties aids decision making regarding reoperation.

Tool 4: For Non palpable Breast Lesions, the Use of Radioactive Seeds, Intraoperative US, or Wire Localization to Direct Lesion Excision is Recommended

A localization method should be used for resection of all non-palpable cancers. Although some studies have indicated superiority of one technique compared with another. Surgeon use of US also aids in targeting and to decide volume of resection in both palpable and non-palpable lesions. Placement of multiple localizing wires or seeds (bracketing) may be useful for larger lesions, multifocal tumors, or extensive ductal carcinoma in situ (DCIS).

Tool 5: Oncoplastic Techniques can reduce the Need for Reoperation in Anatomically Suitable Patients

Oncoplastic techniques have the potential to decrease positive margins at initial lumpectomy by allowing resection of a larger volume of tissue. They also may improve ipsilateral breast appearance and contralateral breast symmetry. It is recommended for only in a selective group of patients. Small primary cancers can be excised with acceptable cosmetic outcome without oncoplastic techniques. For all procedures, marker clips should be placed for post-operative radiation planning.

\section{Tool 6: Specimen Orientation of 3 or More Margins}

When the breast cancer is excised, markers or ink should be placed on the specimen for orientation to ensure which margin edge(s) is/are positive to guide focused re excision of the correct tissue, if necessary. All excisions should be oriented. Orientation is associated with better cosmetic outcomes. The orientation of at least three sides is superior to two sides.

\section{Tool 7: Specimen Radiograph with Surgeon Intraoperative Review}

The primary role of specimen imaging is to document removal of the targeted non palpable lesion before the patient leaves the operating room. Lower-level evidence supports specimen radiography as a method to assess distance of lesion to margin and therefore direct and potentially reduce reoperation. Specimens should not undergo compression during imaging, because it may cause specimen fracture that allows ink to enter the crevasse and a false-positive margin. Surgeons should review the specimen imaging before the operation has been completed, 
ideally with surgeon-radiology communication. Real-time review may avoid a complete "miss" of the lesion or direct the surgeon to perform an additional cavity shave for a "close" margin. Specimen imaging may not be universally available. If not surgeon must develop necessary resources for specimen imaging with immediate image review. Two views at orthogonal angles may identify close or positive margins not seen on a single view.

Tool 8: Consider Cavity Shave Margins in Patients with T2 or Greater Tumor Size or TI with Extensive Intraductal Carcinoma (EIC)

There are moderate levels of evidence that cavity side wall excisions correlate with lower reoperation rate. Shave size should provide adequate sampling of the residual wall. "Tiny shaves" representing only a small portion of a "wall" is discouraged. If shaves are performed, the "final" edge should be marked; i.e., non-oriented shave with even a small amount of tumor on the surface would constitute a final ink positive margin status requiring re excision. Some surgeons routinely perform shaves of all cavity side walls regardless of tumor type or size. Selective shaves directed by palpation, imaging, or pathologic specimen examination. In a randomized, controlled trial of cavity shave versus no-shave margins, showed a statistically significant decrease in the reoperation rate for patients undergoing breast conservation surgery.1, 2.

Tool 9: Intraoperative Pathology Assessment of Lumpectomy Margins may Help Decrease Reexcision When Feasible

A systematic literature review has shown that intraoperative margin assessment with frozen histologic section or imprint cytology are associated with lower reoperation rates by allowing intraoperative reexcision of positive margins 4 . Resources and expertise may limit the feasibility of routine intraoperative pathology assessment.

\section{Tool 10: Compliance with the SSO-ASTRO Margin Guideline to Not Routinely Re operate for Close Margins with no Tumor on Ink in Patients with Invasive Cancer}

Compliance with this guideline has the potential to reduce reoperations by $40 \%$.6. The remaining tools are targeted towards reducing ink positive margins at the initial lumpectomy. By meta-analysis, recurrence risk doubles when ink positive margins are not excised. Recurrence is not improved by reoperation if the margin is negative. If ink positive margins occur, the need for reoperation should be evaluated by the treating team in collaboration with the patient ("shared decision making"), providing patients with recurrence risks in absolute percentages for the choices of reoperation or not. As a consequence, some patients may choose not to have reoperation. The margin guideline is applicable to subsets of patients with "bad tumor biology" (triple negative, her 2 positive, high grade), young age, lobular cancer, EIC, or not receiving systemic treatment. There is no proven benefit for reoperation in these patients if they have ink negative margins. If clinical and/or imaging suggest residual adjacent disease, reexcision should be done. Given the lack of consensus regarding acceptable margin width for DCIS, decisions to 
reoperation in these patients optimally involves multidisciplinary discussions with the patient. Until new evidence is available for DCIS, the NCCN guidelines for re operation to be followed if the margin is ink positive or $<1 \mathrm{~mm} .5,6,7,8$.

\section{Tool 11: Routine Breast-Specific Patient Reported Outcome (PRO) Measurement may help to Assess Cosmetic Outcomes When Feasible}

There is limited reporting in the literature of cosmetic and functional outcomes from the patient perspective. Validated PRO tools, such as BREAST-Q@, should be more widely adopted and may aid improvement. New tools need to be developed that decrease the burdens for both providers and patients for reporting.

Performing reoperation does not mean poor quality care. Particularly, omission of reoperation for positive margins is not recommended. Reoperation of a positive margin is quality care and results in lower risk of cancer recurrence.

\section{References}

1.McCahill LE, Single RM, Aiello Bowles EJ, et al. Variability in re excision following breast conservation surgery. JAMA.2012; 307:467-75.

2. Landercasper J, Whitacre E, Degnim AC, Al-Hamadani M. Reasons for re-excision after lumpectomy for breast cancer: insight from the American Society of Breast Surgeons Mastery(SM) database. Ann Surg Oncol. 2014; 21: 3185-91.

3. Jeffrey Landercasper, Deanna Attai, Dunya Atisha, et al .Toolbox to Reduce Lumpectomy Reoperations and Improve Cosmetic Outcome in Breast Cancer Patients: The American Society of Breast Surgeons Consensus Conference. Annals of Surgical Oncology .2015.22-3174-3183.

4. Chagpar AB, Killelea BK, Tsangaris TN, et al. A randomized, controlled trial of cavity shave margins in breast cancer. N Engl J Med. 2015.

5. Esbona K, Li Z, Wilke LG. Intraoperative imprint cytology and frozen section pathology for margin assessment in breast conservation surgery: a systematic review. Ann Surg Oncol. 2012;19: 3236-45.

6. Boughey JC, Hieken TJ, Jakub JW, et al. Impact of analysis of frozen-section margin on reoperation rates in women undergoing lumpectomy for breast cancer: evaluation of the National Surgical Quality Improvement Program data. Surgery. 2014; 156:190-7. 
7. National Comprehensive Cancer Network. 2015 NCCN guidelines for breast cancer. Margin status in DCIS.

https://www.nccn.org/store/login/login.aspx?,http://www.nccn.org/professionals/physician_gls/p df/breast.pdf. Accessed 15 May 2015

8. Stuart J. Schnitt, Meena S. Moran, and Armando E. Giuliano, Loco regional management of breast cancer . Comments and controversies, Lumpectomy Margins for Invasive Breast Cancer and Ductal Carcinoma in Situ: Current Guideline Recommendations, Their Implications, and Impact . J Clin Oncol, ASCO, May 2020.Volume 38, Issue 20

How to Cite this article As: Prof Ravi Kant \& Prof Bina Ravi (2021). Essentials of Breast Lump assessment and Surgery for early breast cancer for Surgeons. National Medicos Organisation Journal (एन एम ओ जर्नल) (ISSN-2348-3806), 15 (1), 37-42. Retrieved from https://nmojournal.org/index.php/nmojournal/index 